Radial and Nonradial Pulsations as Probes of Stellar Physics

ASP Conference Series, Vol. 259, 2002

C. Aerts, T.R. Bedding, \& J. Christensen-Dalsgaard, eds.

\title{
Multiperiodicity in the Line-Profile Variations of the Spectroscopic Binary $\beta$ Cep Star $\lambda$ Scorpii
}

\author{
K. Uytterhoeven, C. Aerts \\ Instituut voor Sterrenkunde, Katholieke Universiteit Leuven, Belgium
}

\begin{abstract}
A study of the rapid variations of the line profiles of $\lambda$ Scorpii leads to the following new result: we find evidence for the presence of at least 5 periodicities in the intrinsic variations of $\lambda$ Scorpii, which seem to be part of an equidistant multiplet. The modes associated to these periodicities are interpreted as non radial pulsation modes.
\end{abstract}

\section{Introduction}

Many B-type variable stars show line-profile variations (LPVs), which can be explained in terms of non radial pulsations (NRP). A study of LPVs can lead to the determination of the pulsation parameters. Such a study becomes complicated when it concerns fast rotating stars in close binaries. The role of binarity and of rotation on the selection on pulsation modes is not yet understood.

We present an analysis of LPVs of the fast rotating, line-profile variable and close binary $\beta$ Cephei star $\lambda$ Scorpii (HD 158926), based on 421 high-resolution and high-signal to noise spectra of the SiIII lines at $\lambda 4552.6, \lambda 4567.8$ and $\lambda 4574.8$ $\AA$. The data were gathered during 8 subsequent nights in July 1997 , with the CAT/CES combination at ESO La Silla, Chile.

\section{Orbital motion and intrinsic variations}

We used the data to refine the 6 day orbital period found by De Mey et al. (1997) to $P_{\text {orb }}=6.26$ days. The eccentricity $e=0.256$ is quite large for such a short orbit (Uytterhoeven et al., 2002).

Secondly, we studied the intrinsic variability of $\lambda$ Scorpii by means of a study of grayscale representations, which show the line-profile variability in time, and by means of several frequency analyses on different quantities derived from the data. All methods pointed towards the presence of several frequencies, which is quite striking since only one (radial) pulsation mode was detected in photometric data so far (Lomb \& Shobbrook, 1975; Watson 1988).

The frequency analysis was performed on the first three velocity moments of the SiIII profiles and leads to the detection of a multiplet. The power spectrum is dominated by the first frequency, detected also by Lomb \& Shobbrook (1975), and its one day aliases. A phase plot of the first moment for this frequency is shown in Fig. 1. Besides this first frequency four additional frequencies with comparable low amplitudes are present, which form with the first frequency a quintuplet. Within our frequency resolution we can speak of an equidistant 


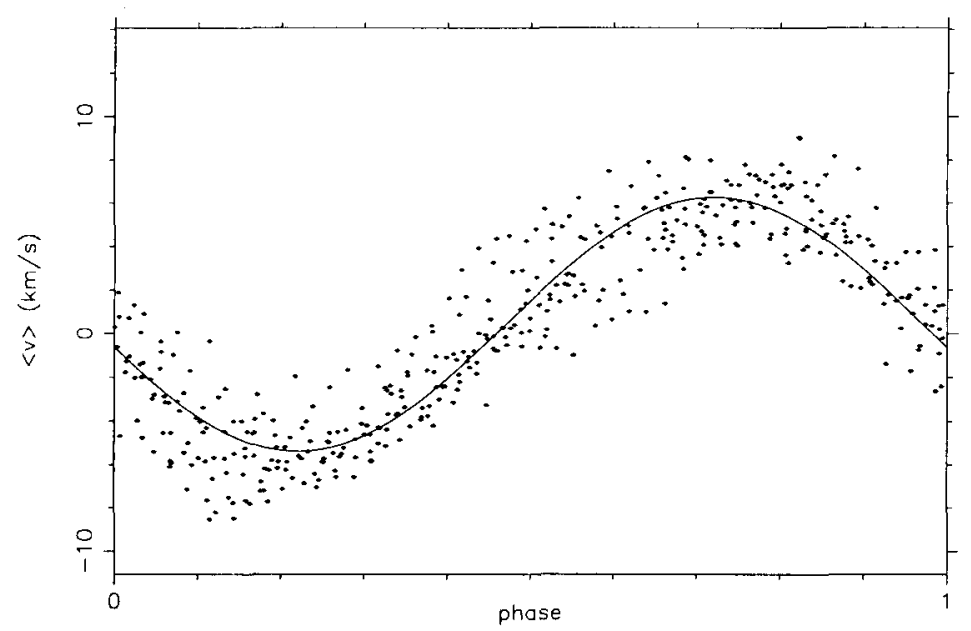

Figure 1. The first velocity moment, calculated from the SiIII line at $\lambda 4552.6 \AA$ folded with the dominant first frequency. The large range of the velocities around this curve is caused by the presence of other frequencies with small amplitudes.

multiplet. In the periodogram we also see four smaller peaks in the noise level which are equally spaced with the five other frequencies. It seems to us more likely that these peaks are induced artificially than that they complete a nonuplet because comparable peaks are visible in the window function (Uytterhoeven et al., 2002).

The presence of a multiplet suggests that rotational splitting is the cause of this frequency structure. In that case a quintuplet is interpreted as caused by $\ell=2$ modes having $m=-2, \ldots, 2$.

\section{References}

De Mey, K., Aerts, C., Waelkens, C., Cramer, S. R., Schrijvers, C., Telting, J. H., Daems, K., \& Meeus, G. 1997, A\&A, 324, 1096

Lomb, N. R., Shobbrook, R.R. 1975, MNRAS, 173, 709

Uytterhoeven, K., Aerts, C., Ausseloos, M. 2002, in ASP Conf. Ser., Observational Aspects of Pulsating B and A stars, eds. C. Sterken \& D. Kurtz, (San Francisco ASP), in press

Watson, R.D. 1988, Ap\&SS, 140, 255 